\title{
An interview with Barbara Fredrickson
}

\author{
University of North Carolina
}

\author{
Interview by \\ Aaron Jarden \\ 4 October 2011
}

\section{What got you interested in wellbeing research?}

I first became interested in studying positive emotions simply because it was an uncharted intellectual frontier when I got into this game twenty years ago. This was back when I was a post-doc in the 1990s. There was an emerging science of emotion and almost everything that was written was about negative emotions, and that just seemed like, well, there must be something interesting about these positive emotions. So that's what bought me there, it was sheer intellectual curiosity to look at what's underneath these stones that have not been picked up.

\section{What do you take "wellbeing" to mean?}

I would say wellbeing encompasses all aspects of health, psychological, physical and social, so it's a very useful aggregate term to capture high functioning or functioning that can be qualified as satisfying or productive on all those different domains.

\section{Why is wellbeing research important?}

I think it's critically important because what we know so far is that the things that predict wellbeing are not the same things that are predicting ill-being or health or disease. If we limited our understanding of wellbeing to just understanding what makes people sick or what leads to psychopathology we'd be missing out on quite a bit of understanding about human nature. Wellbeing needs to be its own target of study because we are uncovering different predictors and different things that help maximise it.

\section{What is the most important application of wellbeing research to our lives?}

I think it's both policy and personal education: that, as individuals, if we share with the public or hear the things that predict and help shape wellbeing, then people can make different choices in their day and their life that can lead them towards wellbeing, since most people intuitively would prefer wellbeing over ill-being. The other very vital application is in terms of policy. Can we reshape communities and institutional norms and cultural norms in ways that aren't continuing to make people sick?

\section{What is the most important wellbeing-related finding from your research?}

What I'm most excited about now is some hints that we are getting in the data that suggest that the particular fusion of the experience of positive emotions in connections with other people 
seems to be driving a lot of the effects. One of the things that we have found is that when people experience positive emotions in their interactions with others, that is predicting improvements in health markers better than other types of social connections and better than other types of positive emotions. When positive emotions are experienced in social connections, that seems to be potentially accounting for the most physical health benefits.

\section{What are you working on right now?}

We are working on a number of different things. One, we are trying to pursue this question of the importance of shared positive emotions more systematically. Another thing that we are looking at is examining how positive emotions lead to changes in gene expression, so we are looking at the effects of positive emotions at a cellular level. We are especially targeting cells related to the immune system's inflammation processes, so this could be one way that we understand the way it is that people's emotional habits translate into wellbeing or ill-being through changes that occur at the cellular level, and that is something that we haven't had access to do before. There are no other studies that I know of that are looking at this in a longitudinal, experimental way. We do a randomised study where one group is randomised into a condition where they learn how to self-generate positive emotions more often compared to another group which learns something totally different that's not connected to positive emotions. We see and track changes in gene expression over one and two years after that to see how those different emotional trainings affect people at the cellular level in ways that prevent illness.

\section{What do you think the next big thing in wellbeing research will be?}

We are going to get better and clearer data that help us understand how emotions and wellbeing are manifested in physical bodies, so that we are beginning to understand that emotions and wellbeing aren't just opinions you have about your life. They're deeply embodied in people's physical responses and cellular structures in ways that allow us to see the substance behind these concepts that we think of as ephemeral and hard to capture. We are going to be able to capture them and give their physiological profile in much more detail and richness than we have in the past. We understand a lot about the physiological profile of stress, and that's because there has been decades upon decades of work on that, and we are getting closer to that level with wellbeing.

\section{What are the main benefits of interdisciplinary research on wellbeing?}

Things that you just couldn't come close to investigating without building interdisciplinary scientific teams. Like the work that my research lab is now engaged in, looking at changes in gene expression, that is actually based on a project that's happening across three different laboratories. There is my lab, that has the expertise in emotions and emotion induction and longitudinal experiments; and we are collaborating with another lab run by Dr. Karen Grewen, where her expertise is in oxytocin and measuring and finding stable measures of that that we can look at over time; and we are also collaborating with Dr. Steve Cole's lab at UCLA. He heads up their social genomics centre. So I don't have to learn from the ground up how to do the social genomics or pieces of it looking at changes in gene expression; instead, I forged a collaboration with someone who has spent twenty years figuring that out, and then he does not have to learn the work on doing experimental interventions to get people to increase their daily doses of positive emotions, we've worked on that for a decade now and so we can join forces 
that way. Interdisciplinary work is challenging, and it's hard to build these teams in ways that are productive and have a mix of rewards, because sometimes people don't know how to credit the work across the different people working on the team. But at one level all those concerns have to be set aside for something to get done, in order for us to get any further, because the kinds of question that we need to address right now demand something larger than one mind, so it demands that interdisciplinary joining of forces.

\section{What would the ideal census question on wellbeing be and why?}

One that I favour is a format that asks you, “Did you laugh yesterday?" So it doesn't ask people to necessarily make a judgement about their life as a whole, but asks them about whether they experienced a particular emotion yesterday. It does not necessarily have to be laughter, it could be, "Did you get to do interesting work yesterday?" or "Did you feel respected yesterday?" I know the Gallup World Poll, based on Daniel Kanehman's input based on his experience with the Day Reconstruction Method, has moved towards that, and I think those more concrete measures of just what your life was like yesterday or today, depending on when you answered the question, can be very useful in getting to the heart of what wellbeing is comprised of, which, in my view, is emotions.

\section{Author}

Aaron Jarden

Open Polytechnic of New Zealand

aaron.jarden@openpolytechnic.ac.nz 